\title{
SMOOTH POLYNOMIAL PATHS WITH NONANALYTIC TANGENTS
}

\author{
ROBERT M. MCLEOD AND GARY H. MEISTERS
}

(Communicated by Paul S. Muhly)

\begin{abstract}
We prove that there exist $C^{\infty}$ functions $\varphi: \mathbf{R}_{t} \times \mathbf{R}_{x} \rightarrow \mathbf{R}$ such that although $\varphi(t, x)$ is a polynomial in $x$ for each $t$ in $\mathbf{R}, \dot{\varphi}(0, x) \equiv(\partial \varphi / \partial t)(0, x)$ need not even be analytic in $x$ let alone polynomial. It was shown earlier by one of the authors [Meisters] that this cannot happen if $\varphi$ satisfies the groupproperty (even locally) of flows, namely if $\varphi(s, \varphi(t, x))=\varphi(s+t, x)$.
\end{abstract}

If a class $C^{1}$ function $\varphi: \mathbf{R} \times \mathbf{R}^{n} \rightarrow \mathbf{R}^{n}$ satisfies the group property

$$
\varphi(s, \varphi(t, x))=\varphi(s+t, x) \quad \forall s, t \in \mathbf{R}, \forall x \in \mathbf{R}^{n}
$$

then it is called a $C^{1}$-flow (in $\mathbf{R}^{n}$ ). If, in addition, it satisfies

$$
\forall t, \quad \varphi(t, x) \text { is a polynomial in } x,
$$

by which we mean that the components of $\varphi(t, x)$ are polynomials in the components $x_{1}, \ldots, x_{n}$ of $x$, then we say that $\varphi$ is a polynomial flow. It is called a smooth polynomial flow if it is also $C^{\infty}$ in $t$. Polynomial flows were conceived in [6], classified (for $n \leq 2$ ) in [1] (up to polyomorphisms of $\mathbf{R}^{2}$ ), and investigated further in [2], [3], [4], [7], and [8]. A diffeomorphism $\rho: \mathbf{R}^{n} \rightarrow \mathbf{R}^{n}$ of $\mathbf{R}^{n}$ into itself for which both $\rho$ and $\rho^{-1}$ are polynomial maps is called a polyomorpnism of $\mathbf{R}^{n}$. If $\varphi(t, x)$ is a polynomial flow in $\mathbf{R}^{n}$, then it follows from (1) that, for each fixed $t$, the mapping $\varphi^{t}$ defined by $\varphi^{t}(x)=\varphi(t, x)$ is a polyomorphism of $\mathbf{R}^{n}$. A function $\varphi$ satisfying (2) can be regarded as a path in the space $\mathscr{P}\left(\mathbf{R}^{n}\right)$ of polynomial maps $\mu: \mathbf{R}^{n} \rightarrow \mathbf{R}^{n}$. The group $\operatorname{GA}\left(\mathbf{R}^{n}\right)$ of all polyomorphisms of $\mathbf{R}^{n}$ is a subspace of $\mathscr{P}\left(\mathbf{R}^{n}\right)$. Those paths in $\mathscr{P}\left(\mathbf{R}^{n}\right)$ which also satisfy (1) are 1-parameter subgroups of $\operatorname{GA}\left(\mathbf{R}^{n}\right)$. The derivative

$$
\dot{\varphi}\left(t_{0}, x\right) \equiv \frac{\partial \varphi}{\partial t}\left(t_{0}, x\right)
$$

could be regarded as the tangent vector to the path $\varphi$ at the point $t_{0}$.

Received by the editors January 27, 1989.

1980 Mathematics Subject Classification (1985 Revision). Primary 26E10; Secondary 14E07.

Key words and phrases. Polyomorphism, polynomial flows, polynomial vector field, smooth $\left(C^{\infty}\right)$ polynomial path, nonanalytic tangent, tangent to path in polynomial space. 
In [1] it was shown that a $C^{1}$ polynomial flow necessarily also satisfies

$$
\dot{\varphi}(0, x) \text { is polynomial in } x
$$

That is, a $C^{1}$ vector field $V: \mathbf{R}^{n} \rightarrow \mathbf{R}^{n}$ which has a polynomial flow $\varphi(t, x)$,

$$
\dot{\varphi}(t, x)=V(\varphi(t, x)), \quad \varphi(0, x)=x,
$$

is necessarily a polynomial vector field: i.e.,

$$
V \in \mathscr{P}\left(\mathbf{R}^{n}\right) \text {. }
$$

This may seem obvious and trivial in the light of the obvious identity

$$
V(x) \equiv \dot{\varphi}(0, x)
$$

which follows from (4) and the property (2) of $\varphi$.

However, as the following theorem shows, without the flow hypothesis (1), $\dot{\varphi}(0, x)$ need not even be analytic in $x$. That is, there are $C^{\infty}$ functions $\varphi$ satisfying (2) for which $\dot{\varphi}(0, x)$ is not analytic at any $x$. That such examples exist was believed by one of us (Meisters) since his paper [6] and earlier. But the discussion of such examples given in [1] is incomplete and inconclusive, while [7, Example 7.1] is only for class $C^{1}$ functions $\varphi$.

Theorem. Let $f$ be a $C^{\infty}$ function on $\mathbf{R}$. Then there is a $C^{\infty}$ function $\varphi$ on $\mathbf{R} \times \mathbf{R}$ such that

1. $\varphi(t, x)$ is a polynomial in $x$ for each $t$,

2. $\dot{\varphi}(0, x)=f(x)$.

( Note that $f(x)$ need not be analytic at any point. See Mandelbrojt [5]. ). Proof. Let $g$ be a $C^{\infty}$ function which vanishes outside $(-1,1)$, is odd, and has $g^{\prime}(0)=1$. Set

$$
a_{n}(t)=g((n+1) t) /(n+1), \quad n=0,1,2, \ldots .
$$

Then the desired $C^{\infty}$ function $\varphi(t, x)$ will be given by the series

$$
\varphi(t, x) \equiv \sum_{n=0}^{\infty} a_{n}(t) P_{n}(x)
$$

for suitably chosen polynomials $P_{n}(x)$ which will be formed by approximating $f(x)$ and its derivatives.

First we use the Weierstrass Approximation Theorem to get polynomials $R_{n}(x)$ such that

$$
\left|f^{(n)}(x)-R_{n}(x)\right|<1 / 2 \quad \text { when }|x| \leq n .
$$

Let $Q_{n}(x)$ be the polynomial such that $Q_{n}^{(n)}(x)=R_{n}(x)$ and $Q_{n}^{(k)}(0)=f^{(k)}(0)$, $k=0,1, \ldots, n$. I.e., $Q_{n}(x)$ is an $n$-fold antiderivative of $R_{n}(x)$.

We will use the following result which follows from Taylor's formula with remainder. 
Lemma. Suppose $\left|h^{(n)}(x)\right| \leq M$ for $|x| \leq b$ and $h^{(k)}(0)=0$ for $k=0,1,2 \ldots$, $n-1$. Then $\left|h^{(k)}(x)\right| \leq M|x|^{n-k} /(n-k)$ ! when $|x| \leq b$ and $k=0,1, \ldots, n$.

Applying this lemma to $f(x)-Q_{n}(x)$, we conclude that

$$
\left|f^{(k)}(x)-Q_{n}^{(k)}(x)\right| \leq|x|^{n-k} / 2(n-k) !
$$

when $|x| \leq n$ and $k=0,1, \ldots, n$. Now let $P_{0}(x)=Q_{0}(x)$ and

$$
P_{n}(x)=Q_{n}(x)-Q_{n-1}(x) \quad \text { for } n \geq 1
$$

From (8) we obtain the following estimates on the derivatives of these polynomials.

$$
\left|P_{n}^{(k)}(x)\right| \leq|x|^{n-k} /(n-1-k) !
$$

when $|x| \leq n$ and $0 \leq k \leq n-1$.

In order to show that $\varphi(t, x)$, given by (7), is $C^{\infty}$ it suffices to show that

$$
\sum_{n=0}^{\infty} a_{n}^{(i)}(t) P_{n}^{(k)}(x)
$$

converges uniformly in each strip $|x| \leq b$. Note first that

$$
a_{n}^{(j)}(t)=(n+1)^{j-1} g^{(j)}((n+1) t)
$$

and thus $\left|a_{n}^{(j)}(t)\right| \leq(n+1)^{j} G_{j}$ where $G_{j}$ is a bound on $\left|g^{(j)}\right|$. Now, from this inequality and (10),

$$
\left|a_{n}^{(j)}(t) P_{n}^{(k)}(x)\right| \leq(n+1)^{j} G_{j} b^{n-1-k} /(n-1-k) !
$$

when $|x| \leq b, n \geq b$, and $n>k$. The ratio test shows that

$$
\sum_{n}(n+1)^{j} b^{n} /(n-1-k) \text { ! }
$$

is convergent.

Thus

$$
\frac{\partial^{j+k}}{\partial t^{j} \partial x^{k}} \varphi(t, x)=\sum_{n=0}^{\infty} a_{n}^{(j)}(t) P_{n}^{(k)}(x),
$$

and since the series is a uniformly convergent series of $C^{\infty}$ functions, $\varphi(t, x)$ is also $C^{\infty}$ on $\mathbf{R} \times \mathbf{R}$.

\section{REFERENCES}

1. H. Bass and G. H. Meisters, Polynomial flow's in the plane, Adv. in Math. 55 (1985), 173-208.

2. B. Coomes, Polynomial flow's, symmetry groups, and conditions sufficient for injectivity of maps, doctoral thesis, University of Nebraska, 1988.

3. __ The Lorenz system does not have a polynomial flow, J. Differential Equations, (to appear).

4. __ Polynomial flow's on $\mathbf{C}^{n}$, (to appear).

5. S. Mandelbrojt, Analytic functions and classes of infinitely differentiable functions, The Rice Institute Phamphlet, Vol. XXIX, no. 1, pp. 2-3, January 1942. 
6. G. H. Meisters, Jacobian problems in differential equations and algebraic geometry, Rocky Mountain J. Math. 12 (1982), 679-705.

7. __ Polynomial flows on $\mathbf{R}^{n}$, Banach Center Publications (Volume on the Dynamical Systems Semester held at the Stefan Banach International Mathematical Center, $\mathrm{u} \ell$. Mokotowska 25, Warszawa Poland, Autumn 1986), (to appear).

8. G. H. Meisters and C. Olech, A poly-flow formulation of the Jacobian conjecture, Bull. of the Polish Academy of Sciences Mathematics 35 (1987), pp. 725-731.

Department of Mathematics, Kenyon College, Gambier, Ohio 43022

Department of Mathematics and Statistics, University of Nebraska-Lincoln, LinColN, Nebraska 68588 